The function $\phi_{l}^{ \pm}(\omega)$ in the last integral of (3.8) is also given by $I_{l 3}{ }^{ \pm}$, where $A_{3}$ has been replaced by $A_{3}{ }^{ \pm}$. If the integration around the circle $S$ follows the external lines in Fig. 1 the + sign must be taken above and the - sign below the real axis.

The method described in this section applies equally to process II and pion-nucleon scattering. But then the functions $\varphi_{0}^{ \pm}$( $S$ and $P_{\frac{1}{2}}$ waves) have a pole at $W=0$, and an arbitrary parameter, the $S$-wave scattering length, is conveniently introduced by means of a subtraction at $\omega=m M$, where the $P_{\frac{1}{2}}$ scattering amplitude vanishes. The fortuitous absence of this pole in $K$-nucleon scattering is due to conservation of strangeness.

\section{FINAL REMARKS}

The objective of setting up dispersion relations for partial amplitudes was to establish an integral representation of functions of a single variable $f_{l}^{ \pm}(\omega)$, for which the unitarity condition takes on, in the physical region, the simple form:

$$
\operatorname{Im} f_{l}(\omega)=\sum_{n} \rho_{n}\left|f_{l}{ }^{n}\right|^{2},
$$

where the index $n$ refers to all allowed channels, compatible with conservation of energy and $\rho_{n}$ is a phase space factor; in the elastic channel $\rho_{n}=k$.

In our integral representation there exist, however, regions of frequencies where the unitarity condition has no simple form. If we attempt to apply the unitarity condition for all $\omega^{\prime}$ in order to obtain an integral equation, we find that the equations for partial amplitudes of different angular momentum and corresponding to processes I, II, and III, in different states of isotopic spin are all coupled. Moreover, in the region $\omega^{\prime}<\omega_{c}$ and along the circle $S$ the unitarity condition can only be obtained by means of analytical continuation.

In the interval $\omega_{c}<\omega<-m M$ the variables $s$ and $t$ in (3.10) are in the physical region for process II. One can re-express $U_{2}$ and $V_{2}$ in terms of the imaginary parts of the scattering amplitudes and expand in partial waves. This expansion is also valid in the unphysical region $-m M<\omega<-\bar{\omega}_{0}$, that is $\left(M_{Y}+\mu\right)^{2}<\bar{s}$ $<(M+m)^{2}$. Only the virtual states $(Y+\pi)$ and $(\Lambda+2 \pi)$ contribute to the amplitudes in this region, and we conjecture that the unitarity condition takes the form (3.13) except for a factor $(-1)^{l}$.

We remark that if all graphs in perturbation theory involving closed baryon loops and four-meson primary interactions are neglected, then from Eq. (3.5) only a cut along $\left(-\infty, \omega_{c}\right)$ survives. However, there is no reason to believe that those graphs are unimportant. It seems, therefore, that in order to make use of these relations as dynamical equations one has to introduce some approximations, replacing the integrals in the unphysical regions by simplified expressions.

\section{ACKNOWLEDGMENTS}

The author expresses his gratitude to Professor R. E. Peierls for his interest and continual support in this work. He is also very much indebted to L. Castillejo for enlightening dicussions and most helpful criticism.

\title{
Completion and Embedding of the Schwarzschild Solution
}

\author{
C. FRONSDAL \\ Theoretical Study Division, CERN, Geneva
}

(Received June 9, 1959)

\begin{abstract}
An analytic manifold is found, the most important properties of which are that it is complete and that it contains the manifold of the Schwarzschild line element. It is thus the complete analytic extension of the latter. The manifold is represented as a Riemannian surface in a six-dimensional pseudo-Euclidean space. The subspace $d \varphi=d \vartheta=0$ is visualized as a two-dimensional Riemannian surface in a 3-dimensional hyperplane in the six-dimensional space. Although the manifold admits groups of motion isomorphic to the real 3-dimensional rotation group and the one-dimensional translation group, it is impossible to introduce a global time-coordinate in such a way that the latter is realized as translations in time. Hence in any global set of coordinates the gravitational field is nonstationary, although it can be made stationary for $r>1$ to any desired approximation. The question of what happens to small test bodies reaching the Schwarzschild critical radius is discussed.
\end{abstract}

STATEMENT OF THE PROBLEM

T

$d s^{2}=\left(1-\frac{1}{r}\right) d t^{2}-\left(1-\frac{1}{r}\right)^{-1} d r^{2}-r^{2}\left(d \vartheta^{2}+\sin ^{2} \vartheta d \varphi^{2}\right)$

1 For a derivation see, e.g., H. Weyl, Space, Time, and Matter (Dover Publications, New York, 1950), p. 252. We have put $2 m \kappa=1$, where $m$ is the mass and $\kappa$ is the gravitational constant.
The coefficient of $d r^{2}$ becomes singular at $r=1$, but it has long been known that this is due only to the choice of coordinates. The proof of this statement may be based on the fact that the Petrov curvature scalars have no singularity at $r=1,{ }^{2}$ but it is perhaps more convincing to refer to the fact that the equations for the geodesics show a singular behavior only at $r=0$. For a motion in

${ }^{2}$ D. Finkelstein, Phys. Rev. 110, 965 (1959). 
the plane $\vartheta=\pi / 2$, we have, using a dot to denote differentiation with respect to proper time ${ }^{3}$ :

$$
\begin{aligned}
\dot{r}^{2} & =k^{2}-1+\frac{1}{r}-\frac{h^{2}}{r^{2}}+\frac{h^{2}}{r^{3}}, \\
r^{2} \dot{\varphi} & =h .
\end{aligned}
$$

Here $k^{2}$ is essentially the energy, and $h$ the angular momentum, of a small test body moving along the geodesic. It is noted that the smallest value of $r$ at which $\dot{r}$ can vanish when $k$ is real is 1 . Hence any geodesic which is not entirely outside $r=1$ must begin or end (or both) at the origin. Equation (2) shows that a small test body reaching the origin from a finite distance does so in a finite proper time. The relation between the Schwarzschild time $t$ and the proper time $\tau$ is given by ${ }^{4}$

$$
(1-1 / r) \dot{t}^{2}=(1-1 / r)^{-1} \dot{r}^{2}+r^{2} \dot{\varphi}^{2}+\epsilon,
$$

where $\epsilon=+1(-1)$ for a time-like (space-like) geodesic and $\epsilon=0$ for a null geodesic. Equation (4) shows that $t \rightarrow \pm \infty$ for a motion along a geodesic approaching $r=1$. Hence it is impossible to describe such geodesics in terms of functions $r(\tau)$ and $t(\tau)$. This state of affairs is expressed by the statement that the coordinate system in which the Schwarzschild line element takes the form (1) is incomplete. The Schwarzschild manifold exists for $0<r<\infty$, but the coordinate patch extends over the interval $1<r<\infty$ only. ${ }^{5}$

The aim of our work is to exhibit a completion of the manifold defined by (1) for $r>1$. It will then be possible to show all possible geodesics in one single picture. This was also the program of a recent effort by Finkelstein. ${ }^{2}$ The relation of our manifold to that of Finkelstein will be shown.

Our method is to avoid the use of coordinates by embedding space-time in a pseudo-Euclidean space of 6 dimensions. One of the purposes of this note is to demonstrate the usefulness of this method in general relativity.

\section{EMBEDDING OF $\mathrm{V}_{4}$ IN $S_{6}$}

The possibility of embedding the line element (1) for $r>1$ in a flat space of six dimensions was first demonstrated by Kasner, ${ }^{6}$ who also showed that an embedding is 5 dimensions is impossible. ${ }^{7}$ Kasner's embedding is given by

$d s^{2}=d Z_{1}^{2}+d Z_{2}^{2}-d Z_{3}^{2}-d Z_{4}^{2}-d Z_{5}^{2}-d Z_{6}{ }^{2}$,

$Z_{1}=(1-1 / r)^{\frac{1}{2}} \cos t, \quad Z_{2}=(1-1 / r)^{\frac{1}{2}} \sin t, \quad Z_{3}=f(r),(6)$

$Z_{4}=r \sin \vartheta \sin \varphi, \quad Z_{5}=r \sin \vartheta \cos \varphi, \quad Z_{6}=r \cos \vartheta$,

\footnotetext{
${ }^{3}$ See, e.g., reference 1, p. 256.

${ }^{4}$ Equation (4) is just the normalization of the proper time interval, $d s^{2} / d \tau^{2}=\epsilon=g_{i j} \dot{x}^{i} \dot{x}^{j}$

${ }^{5} \mathrm{~A}$ familiar instance of the use of an incomplete coordinate system is the "paradox" of Achilles and the Tortoise.

${ }_{6}^{6}$ E. Kasner, Am. J. Math. 43, 130 (1921).

${ }^{7}$ E. Kasner, Am. J. Math. 43, 126 (1921).
}

where

$$
(d f / d r)^{2}=\left(\frac{1}{4 r^{3}}+1\right)(r-1)^{-1} .
$$

If one tries to eliminate the coordinates, Eq. (8) must be solved for $r$ as a function of $f=Z_{3}$. This is possible only for $r>1$. Hence this embedding may not be extended to $r<1$. Another shortcoming of (6), (7) is that $Z_{1}$ and $Z_{2}$ are periodic functions of $t$, so that the embedding identifies distinct points of the original manifold. This suggests replacing the trigonometric functions by hyperbolic functions. If we write

$$
\begin{gathered}
d s^{2}=d Z_{1}{ }^{2}-d Z_{2}^{2}-d Z_{3}{ }^{2}-d Z_{4}{ }^{2}-d Z_{5}{ }^{2}-d Z_{6}{ }^{2}, \\
Z_{1}=2(1-1 / r)^{\frac{1}{2}} \sinh (t / 2), \\
Z_{2}=2(1-1 / r)^{\frac{1}{2}} \cosh (t / 2), \\
Z_{3}=g(r), \quad Z_{4}=r \sin \vartheta \sin \varphi, \cdots,
\end{gathered}
$$

we find

$$
(d g / d r)^{2}=\left(r^{2}+r+1\right) / r^{3} .
$$

It is now possible to eliminate the coordinates, but it is simpler to eliminate $t, \vartheta$, and $\varphi$ only. We get

$$
\begin{aligned}
Z_{2} & =+\left(4-\frac{4}{r}+Z_{1}^{2}\right)^{\frac{1}{2}}, \\
Z_{3} & =+\int d r\left(r^{2}+r+1\right)^{\frac{1}{2}} / r^{\frac{3}{2}}, \\
Z_{4}{ }^{2}+Z_{5}^{2}+Z_{6}{ }^{2} & =r^{2} .
\end{aligned}
$$

In principle (14) may be solved for $r\left(Z_{3}\right)$ and the result used to eliminate $r$ from (13) and (15), but it is simpler, and quite harmless, ${ }^{8}$ to retain $r$ as a parameter. The surface defined by (13)-(15) is analytic not only in the original interval $1<r<\infty$, but in the whole interval $0<r<\infty$. Hence it is an analytic continuation of the Schwarzschild line element. It is still not complete, since it will become clear later that there are geodesics which pass through $Z_{2}=0$. But if we adjoin the other branch of (13) we get a manifold which is complete and analytic everywhere except at $r=0$. Hence the analytic completion of the Schwarzschild line element is represented as the surface

$$
\begin{aligned}
Z_{2}^{2}-Z_{1}^{2} & =4(1-1 / r), \\
Z_{3} & =+\int d r\left(r^{2}+r+1\right) r^{-\frac{3}{2}}, \\
Z_{4}^{2}+Z_{5}^{2}+Z_{6}^{2} & =r^{2} .
\end{aligned}
$$

In addition to completeness and analyticity we note the following properties of the surface (16). It admits a group of motions which is isomorphic to the real rotation group (rotations in the $Z_{4}, Z_{5}, Z_{6}$ hyperplane), as well

\footnotetext{
${ }^{8}$ Because $Z_{3}$ is a monotonous function of $r$.
} 


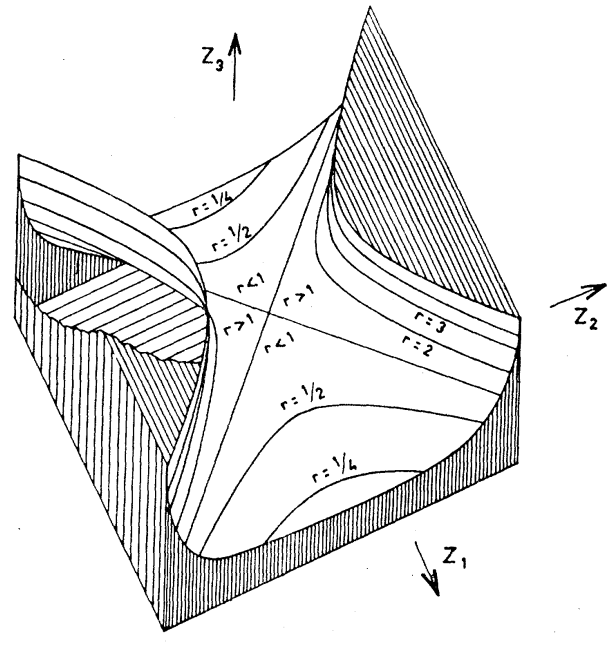

Fig. 1. The surface defined by Eqs. (18), showing the subspace $d \vartheta=d \varphi=0$ of the completed Schwarzschild manifold as a 2dimensional surface in a pseudo-Euclidean space. Of the coordinate directions $Z_{1}$ is time-like, $Z_{2}$ and $Z_{3}$ space-like.

as a one-parameter translation group (imaginary rotations in the $Z_{1}, Z_{2}$ plane). The existence of the latter corresponds to the stationary character of (1). It is also invariant under space reflection $\left(Z_{4}, Z_{5}, Z_{6} \rightarrow-Z_{4}\right.$, $\left.-Z_{5},-Z_{6}\right)$, under time reflection $\left(Z_{1} \rightarrow-Z_{1}\right)$, and under the reflection $Z_{2} \rightarrow-Z_{2}$. [Of course it is possible to square (14) to obtain an additional reflection invariance, but this is meaningless since there are no geodesics connecting $d Z_{3} / d r>0$ and $d Z_{3} / d r<0$.] The manifold (16) contains two Schwarzschild inside regions and two outside regions. Time-like geodesics connect the two inside regions, but not the two outside ones.

\section{THE SUBSPACE $d \boldsymbol{\vartheta}=d \varphi=0$}

For the further investigation of the complete manifold (16) it is convenient to be able to draw a picture of the manifold. We therefore specialize to the subspace $d \vartheta=d \varphi=0$. This is entirely sufficient for discussing the penetration of geodesics into the interior. The line element is then

where

$$
\begin{aligned}
d s^{2} & =(1-1 / r) d t^{2}-(1-1 / r)^{-1} d r^{2} \\
& =d Z_{1}{ }^{2}-d Z_{2}{ }^{2}-d Z_{3}{ }^{2},
\end{aligned}
$$

$$
\begin{aligned}
Z_{2}{ }^{2}-Z_{1}{ }^{2} & =4(1-1 / r), \\
Z_{3} & =\int d r\left(r^{3}+r^{2}+r+1\right)^{\frac{1}{2} r^{-\frac{3}{2}}} .
\end{aligned}
$$

This surface is shown in Fig. 1. The lines of constant $r$ are also lines of constant $Z_{3}$, and are approximately equidistant in the $Z_{3}$ (vertical) direction. The two separate parts of the surface for which $r<1(r>1)$ are two Schwarzschild interiors (exteriors).

Figure 2 shows the projection of the surface in the $Z_{1}, Z_{2}$ plane. Some light cones are shown. They may be determined quite simply from

$$
\begin{gathered}
d Z_{1}^{2}-d Z_{2}^{2}=d Z_{3}^{2}=\left(r^{3}+r^{2}+r+1\right) d r^{2} / r^{3}, \\
Z_{2} d Z_{2}-Z_{1} d Z_{1}=2 d r / r^{2}
\end{gathered}
$$

or

$$
\begin{gathered}
d Z_{2} / d Z_{1} \equiv \alpha \\
1-\alpha^{2}=\frac{r\left(r^{4}-1\right)}{4(r-1)}\left(Z_{2} \alpha-Z_{1}\right)^{2}
\end{gathered}
$$

We may now discuss the introduction of coordinates in this subspace. The obvious choice is to take $Z_{1}$ as time coordinate and $Z_{2}$ as space coordinate. This is a perfectly well-behaved set of coordinates from a mathematical point of view. However, one should like to retain $r$ as space coordinate, because the term $r^{2}\left(d \vartheta^{2}+\sin ^{2} \vartheta d \varphi^{2}\right)$ in the line element identifies $r$ as the physical radius. In choosing a time coordinate one is interested in the following features. (a) The coordinate system should be pseudo-Galilean at infinity (i.e., as $r \rightarrow \infty$ ). (b) The metric tensor should be independent of time. The first requirement causes no difficulty in principle, but we shall show that no global time coordinate satisfying (b) exists.

Schwarzschild's time is defined in the top quadrant of Fig. 2 by

$$
e^{t}=\frac{1+Z_{1} / Z_{2}}{1-Z_{1} / Z_{2}} .
$$

We see that $t$ tends to - $-(+)$ infinity near the left (right) line $r=1$. The only redefinition of the time which does not introduce time explicitly into the components of the metric tensor is that obtained by adding a function of $r$ to $t$. Hence we would have the time

$$
e^{T}=\frac{1+Z_{1} / Z_{2}}{1-Z_{1} / Z_{2}} f(r) .
$$

But now it is clear that no choice of $f(r)$ can make $T$ finite both at $Z_{1}=Z_{2}$ and at $Z_{1}=-Z_{2}$, since $r$ and hence

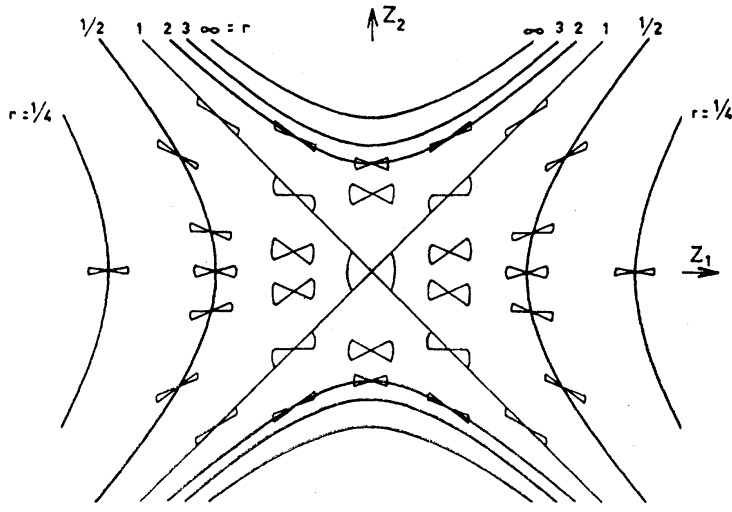

Fig. 2. The projection of the surface in Fig. 1 in the $Z_{1}$, $Z_{2}$ plane. This projection is one-valued. 
$f(r)$ has the same value at $Z_{1}= \pm Z_{2}$. This means that the largest portion of the complete manifold for which a global time can be defined, of which the metric tensor is independent, is one of the halves bounded by either $Z_{1}=Z_{2}$ or $Z_{1}=-Z_{2}$. With the choice $f(r)=(r-1)^{-1}$ we have

$$
e^{T}=\frac{1+Z_{1} / Z_{2}}{1-Z_{1} / Z_{2}} \frac{1}{r-1}
$$

or

$$
T=t-\ln (r-1) \text {. }
$$

This time is that introduced by Finkelstein, ${ }^{2}$ it is a time coordinate for the left and the top quadrants in Fig. 2. It is clear why Finkelstein's coordinate system is incomplete. Geodesics can enter the interior in a finite $T$ only if $d T / d \tau$ is negative.

One may ask whether a definition of global time may be found such that the field is approximately stationary. This may be accomplished for example by taking

$$
e^{T}=\frac{1+Z_{1} / Z_{2}}{1-Z_{1} / Z_{2}} \frac{r^{n}-Z_{1} / Z_{2}}{r^{n}+Z_{1} / Z_{2}} .
$$

By taking $n$ large enough this $T$ may be made to differ arbitrarily little from $t$ when $r>1$. The definition (26) suffers from other shortcomings which may, however, easily be overcome. However, the pursuance of this point is not interesting.

\section{DO GEODESCIS PENETRATE $r=1$ ?}

We know by Eq. (2) that if $\dot{r}(\tau)$ is continuous then $r(\tau)$ passes with no difficulty through the value $r=1$, and in Fig. 2 we may draw such geodesics and see that the impossibility of drawing them in the $r, t$ plane is due only to an unfortunate choice of time coordinate. It is possible to draw a static picture of the Schwarzschild world consisting of two outside regions joined at $r=1$ and no inside ones. ${ }^{9}$ There are also embeddings of space-time which end at $r=1$, notably the Kasner embedding. ${ }^{6}$ Other embeddings which end at $r=1$ have been found by Fierz. ${ }^{10}$ These surfaces are perfectly continuous and geodesics reaching $r=1$ apparently pass into the other world. It is only by demanding that $\dot{r}$ be continuous at $r=1$ that our interpretation becomes the only one possible. However, since no correlation exists between geodesics going in through $r=1$ and those coming out, the question is meaningless from a physical standpoint. The reason why there can be no correlation between outgoing and ingoing geodesics is that all geodesics going in (out) end (begin) at $r=0$. Hence, contrary to the situation of classical mechanics in which the last term in (2) is absent, the geodesics that reach $r=0$ are not proper limits of geodesics that do not. Finally, it may be pointed out that the singular character of the surface $r=1$ is a feature intimately connected with the nondefiniteness of the space-time metric. Because of the need to choose a six-space signature with at least one plus sign, the concept of nearness is not immediately connected with the line element. The lines $r=1$ are, in fact, always lines of zero length.

\section{ACKNOWLEDGMENTS}

It is a pleasure to acknowledge the hospitality extended to me at CERN, and particularly the generous help and advice given me by Professor M. Fierz in the course of this work.

Note added to proof.-Professors D. Finkelstein and J. A. Wheeler have kindly informed me that Professor M. Kruskal at Princeton University has obtained the complete manifold some time ago (unpublished). Kruskal's time and radial coordinates are similar to our $Z_{1}$ and $Z_{2}$.

${ }^{9}$ This is the Flamm paraboloid [L. Flamm, Physik. Z. 17, 448 (1916)].

${ }^{10} \mathrm{M}$. Fierz (private communication). 